\title{
Structural variability and luminescence color tuning in lanthanide-organic hybrid materials
}

\author{
R. Lee Ayscue III, Chloe P. Verwiel, and Karah E. Knope*
}

Georgetown University, Department of Chemistry, $37^{\text {th }}$ and O St, Washington, DC 20057, USA rla41@georgetown.edu

Luminescent materials are found in a variety of applications including lighting, electronic displays, and even sensor technologies. One strategy for imparting luminescence to a material is through the incorporation of lanthanides which display strong visible- and/or NIR- light emission upon proper sensitization by light harvesting ligands. A second design strategy is the incorporation of organic molecules that can exhibit broad visible emission. Targeting both strategies, this work examines novel lanthanide-organic hybrid materials that consist of molecular complexes of La, $\mathrm{Eu}, \mathrm{Gd}$, and/or $\mathrm{Tb}$ metals with phenanthroline and thiophene carboxylate organic ligands. Analysis of structural features, such as structure type, intermolecular packing interactions, and the degree of metal ion site substitution affords valuable insight into their influence over the energetic transitions that impart observed luminescent behaviors in the solid state. These behaviors include: extension of the excitation manifold into the visible region, dual emission originating from ligand and lanthanide components, and subsequent luminescence color tuning dependent not only on Ln doping percentages but also excitation wavelength. Using X-ray diffraction and solid-state photoluminescent techniques, this work aims to investigate and correlate fundamental structure-property relationships that are appreciable for future materials design.

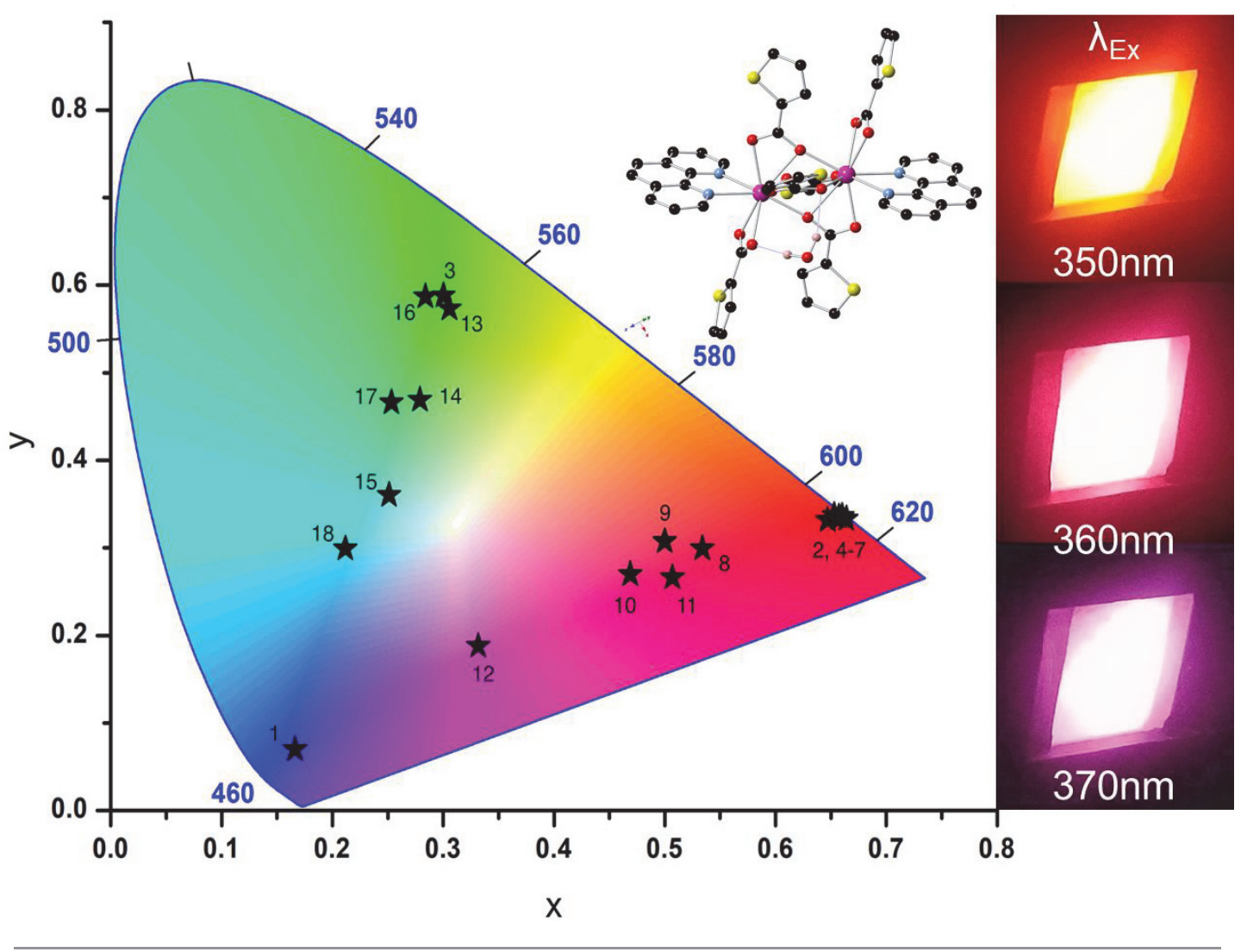

